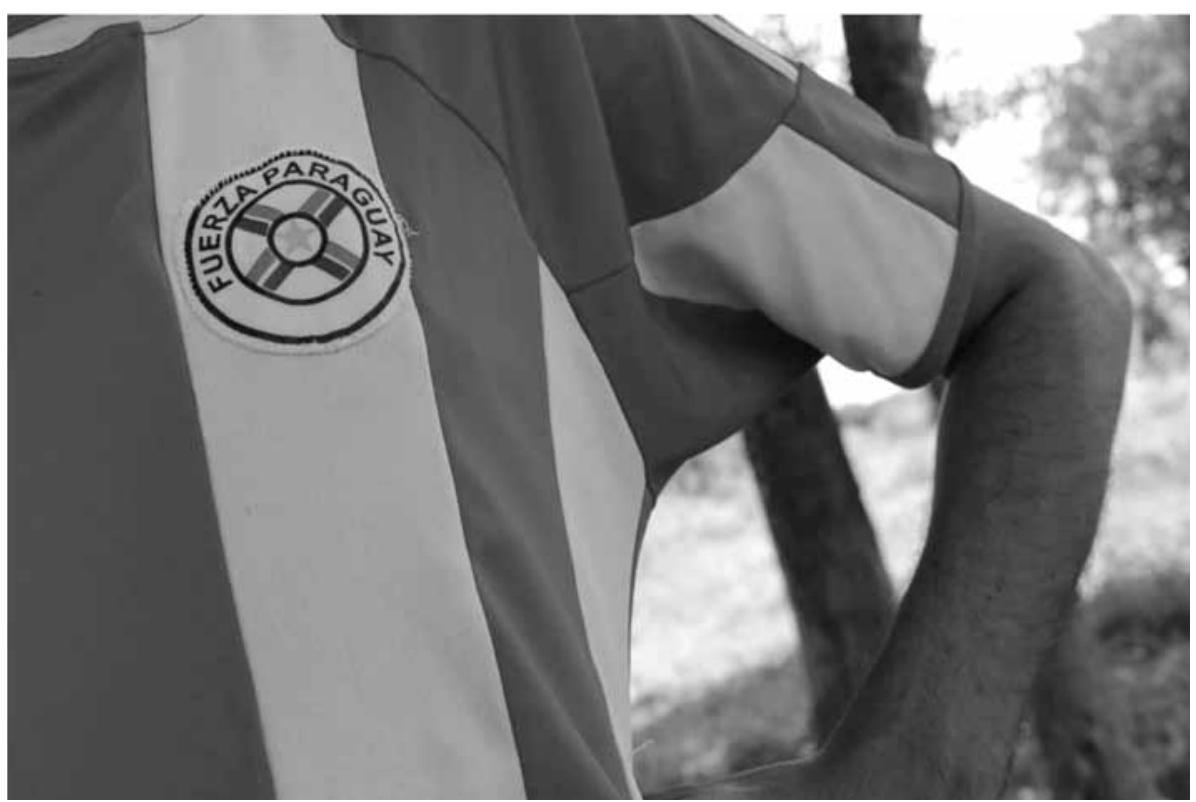

Foto: Carlos Freire da Silva

Detalle de paraguayo. Repatriación, Caaguazú.

\title{
Los migrantes paraguayos y la lengua guaraní
}

\author{
Miguel Ángel Verón *
}

Cuando viajé en el 2011 a Minas Gerais para defender mi tesis en un curso de post grado que cursé en la Universidad Federal de Juiz de Fora, en la ciudad del mismo nombre, abordé primero un ómnibus que me llevara de Asunción a San Pablo, y de esta ciudad otro que me trasladara a la histórica ciudad de Juiz de Fora. En el primer ómnibus, me llamó la atención la cantidad de jóvenes que nos acompañaban. Muy pronto distinguí entre ellos dos grupos: uno,

\footnotetext{
* Lingüísta, Director General de Planificación Lingüística de la Secretaría de Políticas

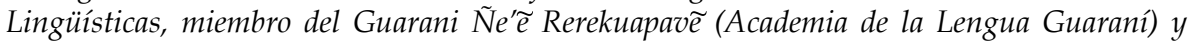
Profesor de la Universidad Nacional de Asunción.
} 
conformado solamente de mujeres, todas señoritas jóvenes, y otro, integrado de hombres y mujeres. Como siempre vengo haciendo desde hace unos años, me acerqué a ambos grupos para escuchar qué lengua utilizaban y de qué clase social provenían. Enseguida pude constatar que las integrantes del primer grupo hablaban castellano, eran de Asunción, e iban a San Pablo a pasar unos días de vacaciones. Todas ellas eran estudiantes universitarias. Enseguida descubrí que los otros jóvenes, tres mujeres y cuatro hombres, hablaban guaraní, eran del departamento de Caaguazú y residían en la ciudad paulista, donde trabajaban como costureros. Dado que soy de tierra adentro y de origen y principio proletario, prontamente entramos en sintonía con estos jóvenes trabajadores con quienes conversé distendidamente. Gracias a las conversaciones, descubrí un fenómeno social nuevo para mí: el éxodo de los jóvenes de mi querido departamento, Caaguazú, a la colosal ciudad de San Pablo. Descubrí, al mismo tiempo, que la ciudad receptora de los jóvenes paraguayos de este departamento, expulsados del campo, ya no era la ciudad bonaerense de otras épocas, sino la ciudad que en épocas lejanas fuera del dominio de los Ñe'ẽngatu.

Detrás de mis curiosidades lingüísticas, pude descubrir muchos fenómenos socioeconómicos nuevos que se sucedían en el quinto departamento, y en gran medida en todo el campo paraguayo. Percibí que lamentablemente los jóvenes campesinos seguían siendo obligados por las crisis económicas a migrar a las urbes más grandes del país o a otros países de la región, que los jóvenes del departamento de Caaguazú ya no migraban solamente a Buenos Aires, como lo hicieron mis hermanos hace 20 a 30 años atrás, sino a la ciudad de San Pablo; que los jóvenes que trabajan en la ciudad paulista se dedicaban preferentemente al rubro de la confección de prendas de vestir, que los mismos eran el sostén de sus padres y de sus hermanos y hermanas menores que quedaban en el campo, $y$, por último, y es lo que más interesa en este artículo, que los jóvenes campesinos seguían manteniendo su lengua materna, el guaraní, más allá de las fronteras paraguayas.

\section{Situación sociolingüística del Paraguay}

El Paraguay es un crisol lingüístico donde conviven varias lenguas. Además de los dos idiomas oficiales, guaraní y castellano, y las 19 lenguas que hablan los pueblos indígenas, son habladas varias otras en las comunidades de inmigrantes que por suerte mantienen sus lenguas y contribuyen para la consolidación del multilingüismo paraguayo.

Según el Censo Nacional de Población y Vivienda de $2002^{1}$, en el Paraguay el guaraní es el idioma más utilizado por la población paraguaya, seguido del castellano. El mencionado censo arrojó los siguientes resultados (MELIÀ, 2010, p. 162): 


\begin{tabular}{lrl}
\hline \multicolumn{1}{c}{ Lengua } & Populación & (\%) \\
\hline Guaraní parlantes & 1.399 .220 & 27,0 \\
Guarani bilingües & 1.721 .200 & 33,0 \\
Castellano bilingües & 1.330 .810 & 26,0 \\
Castellano parlantes & 411.780 & 8,0 \\
Idioma portugués & 122.520 & 2,4 \\
Idioma alemán & 36.200 & 0,7 \\
Idioma japonés & 3.210 & 0,1 \\
Idioma coreano & 2.810 & 0,1 \\
Pueblos indígenas & 87.099 & 1,8 \\
Otros idiomas no indigenas & 3.960 & 0,1 \\
\hline
\end{tabular}

Según los estudios de Melià, de estas cifras resulta, respecto al bilingüismo guaraní castellano, el siguiente cuadro:

\begin{tabular}{lrr}
\hline \multicolumn{1}{c}{ Lengua } & Populación & (\%) \\
\hline Guaraní & 3.120 .420 & 60,0 \\
Bilingüe (guaraní-castellano) & 3.052 .010 & 59,0 \\
Bilingüe (castellano-guaraní) & 1.330 .810 & 26,0 \\
Castellano & 411.780 & 8,0 \\
\hline
\end{tabular}

\section{La lengua guaraní}

La lengua guaraní es una de las lenguas indígenas más vigorosas del continente. A la llegada de los conquistadores, era el idioma de mayor prestigio y de uso más extendido en vastas regiones del continente americano. Las abundantes toponimias testimonian que esta lengua se utilizaba en los territorios que hoy ocupan Paraguay, Brasil, Argentina, Uruguay, Ecuador, Colombia y otros países del continente:

En la época del descubrimiento y de la conquista, la llamada lengua guaraní-tupí estaba muy difundida en América del Sur, particularmente en los territorios que actualmente comprenden Paraguay, Brasil, Argentina, Bolivia y Uruguay y, en menor grado, Perú, Ecuador, Guayanas. Eran tierras pobladas por guaraníes y por otros pueblos de diferentes lenguas pero que aceptaban el guaraní-tupí por gozar de mayor prestigio cultural.

Desde el núcleo central del hábitat de los Guaraní-Tupí la 
lengua se difundió por una extensión mayor que el territorio por ellos ocupado, testimonio de esto es la toponimia en guaraní, prácticamente por toda América del Sur. Era la lengua más extendida en esta parte del continente, al punto que los descubridores y colonizadores que entraban por la costa atlántica la conocían como la "língua mais usada na costa do Brasil", "língua geral" (lengua general) (GONZÁLEZ TORRES, 2007, p. 13).

Durante la colonia, la Corona Española trató de imponer el castellano como lengua única en el Paraguay, como impulsó en los otros países del continente, dado que "la lengua acompaña al imperio", como defendía Antonio de Nebrija. Esta política, empero, no tuvo resultados esperados en Paraguay donde la lengua de Castilla no terminaba de consolidarse. El uso oral y escrito de la lengua guaraní era normal, hasta en la administración del Estado colonial. Los cabildos de los pueblos indígenas se comunicaban en guaraní escrito con los gobernadores y los reyes. Por otra parte, en 1603 la Iglesia Católica resolvió adoptar oficialmente el idioma guaraní para la enseñanza de la doctrina cristiana por ser la lengua más clara y hablarse generalmente en todas estas provincias, siendo su conocimiento obligatorio para los curas encargados de esa enseñanza (ROMERO, 1998, p. 21).

\section{La diglosia paraguaya}

Históricamente el guaraní ha sido la lengua de mayor uso en el Paraguay, pero por rémoras del colonialismo cultural y lingüístico, la misma, ya que es de origen americano, ha sido subvalorada y estigmatizada, y su uso reducido al calor del hogar, a las calles, a la oralidad, a las situaciones informales; mientras el castellano, por ser de origen europeo, y a pesar de ser la lengua minoritaria, fue desde los inicios de la independencia la lengua de la administración del Estado, del sistema educativo, de la formalidad y de la clase dominante, lengua también en que esta ejercía el poder sobre la mayoría de la población de lengua materna guaraní. Esta, a pesar de ser mayoritaria, fue minorizada, y el castellano, a pesar de ser minoritario, mayorizado por el Estado paraguayo. Además, el castellano ha sido la lengua de las ciudades, y el guaraní el idioma del campo, donde hasta unas décadas atrás vivía la mayoría de la población paraguaya, antes del acelerado proceso de descampesinización, producto de la expulsión campesina de parte del agro negocio.

\section{Perfil lingüístico de los compatriotas emigrantes}

Hay muchas razones por las que las personas abandonan su país y emigran a otro, pero la mayoría de las migraciones obedecen a factores económicos. 
En Paraguay, casi la totalidad de los jóvenes que emigran del país para buscar trabajos son campesinos, y la mayoría de los mismos, sino la totalidad, son de lengua materna guaraní. La gran mayoría aprende el castellano ya en el exterior. Los castellano hablantes, y con mayor razón los monolingües en esta lengua, moran en las ciudades grandes, especialmente Asunción, y son de clase económica acomodada. Si migran, normalmente lo hacen para proseguir sus estudios de grado y de pos grado. Los monolingües guaraní tienen poca cabida en las ciudades, donde la lengua dominante es el castellano; y si viven en ellas, se dedican a empleos informales y de baja remuneración. Ellos, en la práctica, no pueden ser funcionarios del Estado, dado que este usa solamente el castellano en todos sus documentos y comunicaciones en general; además, los guaraní hablantes no pueden estudiar en las universidades, puesto que estas utilizan solamente el castellano como lengua de enseñanza.

\section{Lengua que hablan los emigrantes paraguayos}

Los paraguayos de la diáspora mantienen las dos lenguas oficiales de su país: el guaraní y el castellano, pero generalmente se identifican más con la primera, que es la lengua popular paraguaya por antonomasia. Los jóvenes paraguayos que estudian en los diversos países de la región cuentan que utilizan el guaraní como código secreto. En las universidades de Cuba llaman el inglés paraguayo. En la Universidad Federal para la Integración Latinoamericana (UNILA) de Brasil, los paraguayos utilizan el guaraní para comunicarse entre sí y el castellano para interactuar con estudiantes de otros países.

Me contó la profesora María Eta Vieira que le llamaba la atención que muchos estudiantes de esa universidad latinoamericana utilizaran una lengua diferente del portugués y del español; cuando preguntó de qué lengua se trataba, le respondieron que era el guaraní. Este hecho la impulsó a elaborar un proyecto para desarrollar cursos de guaraní para estudiantes y profesores de los distintos países que estudian en esa universidad, empresa en la que la apoyó el profesor Derlis Sandoval, alumno de esa universidad, hoy traductor guaraní-portugués del Parlasur. Los cursos de guaraní iniciaron en setiembre de 2011 y con mucho éxito siguen desarrollándose hasta la fecha.

Me consta también que los paraguayos y las paraguayas residentes en Buenos Aires mantienen el guaraní al lado del castellano. El 10 de mayo de 2010 fuimos invitados cuatro escritores guaraníes a la Feria de Libro de Buenos Aires, que ese año se convirtió en la Capital Mundial del Libro. En la fecha mencionada, los cuatro escritores, además de presentar nuestras obras en la Feria, tuvimos la oportunidad de compartir con los compatriotas en el Deportivo Paraguayo, durante cinco horas, conversando en guaraní, ante una multitudinaria audiencia. Los paraguayos que viven en otros países de América y de otros continentes también siguen hablando las dos lenguas oficiales de nuestro país. 


\section{Los paraguayos emigrados a San Pablo hablan guaraní y portugués}

Los jóvenes paraguayos que por necesidad económica migran a San Pablo para trabajar mantienen la lengua guaraní e ineluctablemente deben aprender y comunicarse en la lengua local: el portugués. Los mismos son braceros, obreros dependientes de la cadena productiva y de explotación local, por ello deben vivir en las culturas y las lenguas locales. Las mismas condiciones de exigencia no encuentran los brasileros que vienen a Paraguay a expulsar a los campesinos e indígenas de sus tierras para la plantación de soja. Estos, a pesar de que vienen a vivir en tierras extranjeras, imponen su lengua como también sus culturas, debido a la endeble posición del Estado paraguayo para defender la soberanía patria.

El lunes 9 de junio del corriente año (2014), bien temprano, nos acercamos con mis compañeros de trabajo a una gasolinera de la ciudad de La Paloma, departamento de Canindeyú. Con mucha gentileza nos atendió un joven de 24 años que hablaba un fluido guaraní. Le pregunté si hablaba portugués además del guaraní, y me respondió afirmativamente; le interrogué, también, si hablaba español, pregunta a la cual me respondió negativamente: “Yma añe'ẽ, ko'ágã ndaikatuvéi añe'ẽ, ojehe'apa chehegui portugués ndive" (Antes lo hablaba, ahora ya no, se me mezcla con el portugués). En su propia tierra este joven, como aquellos que emigran a la capital paulista, es presionado a aprender y comunicarse en la lengua lusitana, hasta tal punto de olvidar el castellano, lengua oficial de su país y segunda lengua suya.

Los colonos brasileros avasallan la confianza y la apertura que les dimos los paraguayos, y antes de aprender y ajustarse a las culturas y lenguas propias de nuestro pueblo, imponen con soberbia su lengua y sus modos de ser y estar en el mundo; el portugués se impone como el monocultivo de soja que carcome el campo paraguayo. Los paraguayos que emigran al hermano país de Brasil no cuentan con los mismos medios de producción, sino son braceros, razón por la cual deben aprender la lengua del país que los acogió, como es natural. De los datos que pude rescatar de los jóvenes con quienes viajé a San Pablo en el 2011, los paraguayos que trabajan en esa ciudad hablan muy poco el castellano; la lengua de la comunicación entre ellos es el guaraní, y el portugués la segunda lengua, idioma que les permite interactuar con la sociedad local.

\section{Lengua que trasmiten los emigrantes paraguayos a sus hijos}

Un fenómeno sociolingüístico que nos preocupa y ocupa a las personas que promovemos la normalización del uso de la lengua guaraní es el peligro del corte generacional en la transmisión de esta lengua. El guaraní ha llegado a este siglo gracias al hogar, a la familia y a pesar del Estado y la escuela. La transmisión de los padres a los hijos es la mayor garantía de la vitalidad presente y futura de una 
lengua. En este momento en Paraguay la mayoría de los papás y mamás bilingües guaraní-castellano ( $60 \%$ de la población) no les pasa el guaraní a sus hijos, sino solamente el castellano, privándoles de esa manera a sus hijos de la posibilidad de aprender y conocer las dos lenguas oficiales de su país desde la cuna. Este mismo fenómeno producido por las fuerzas sociolingüísticas imperantes en el país, se presenta - por lo que he podido indagar - entre los paraguayos y las paraguayas que viven en el exterior. Los mismos siguen hablando el guaraní, pero no lo transmiten a sus hijos.

Hace unos años encontré a una compueblana, amiga de la infancia que vivió y respiró solamente en guaraní hasta que migró a Buenos Aires. Con ella se encontraba su hija de unos cinco años. En medio de la conversación le pregunté si su hija hablaba guaraní: “¿Para qué, en qué le va ayudar el guaraní allá en Buenos Aires?", fue su inmediata respuesta. Traté de persuadirle que era importante que su hija hablara la lengua de la madre; que era importante que su hija sepa comunicarse en la única lengua que hablan y entienden los abuelos, dado que tendría muchos problemas de comunicación las veces que viene a Paraguay para verlos, pero mis argumentos fueron estériles para convencer a esta amiga. Actitudes de subvaloración o rechazo de la lengua propia del Paraguay como las de esta compatriota pululan entre los paraguayos dentro y fuera del país. Pero por suerte desde el Estado y la sociedad se están desarrollando políticas y acciones que apuntan a la superación de este tipo de posiciones alienadas y alienantes.

La Secretaría de Políticas Lingüísticas (SPL) está encarando proyectos para revertir este tipo de actitudes, desarrollando planes y proyectos para elevar el estatus de la lengua guaraní, y de esa manera acrecentar el orgullo y la lealtad de sus hablantes hacia la misma. Gratamente me ha sorprendido que los paraguayos residentes en el exterior estén ocupándose en este momento de que sus hijos no pierdan el guaraní, y desarrollando cursos para ellos. Este tipo de decisiones nos dan la certeza de que con las nuevas políticas lingüísticas desarrolladas en Paraguay estará asegurada la vitalidad futura de la lengua mayoritaria del Paraguay. La Secretaría de Políticas Lingüísticas, convenio de cooperación mediante, está trabajando con la Secretaría de Desarrollo para Repatriados y Refugiados para promover la transmisión intergeneracional y la enseñanza de la lengua guaraní entre los paraguayos de la diáspora. A través de una campaña de donaciones, la SPL juntó setecientos diez (710) textos y diez (10) unidades de materiales audiovisuales en guaraní; dichos textos han sido distribuidos por la Secretaría de Repatriados en varias ciudades de países como Argentina, Ecuador, España, Austria. Solamente en Buenos Aires se están desarrollando en distintos lugares ocho cursos de lengua guaraní para hijos e hijas de paraguayos; los mismos están utilizando los textos recogidos por la SPL y distribuidos por la Secretaría de Repatriados.

Además de eso, la Secretaría de Políticas Lingüísticas, en forma conjunta con la Secretaría Nacional de Tecnologías de la Información y Comunicación (SENATICS), está desarrollando un proyecto sumamente auspicioso y de gran 
envergadura: la elaboración de un curso multimedial de lengua guaraní, con los mismos estándares de calidad que los cursos realizados con algunos otros idiomas de mayor demanda. Con este proyecto habremos tenido como país una herramienta de suma utilidad para la enseñanza y aprendizaje de la lengua guaraní de manera interactiva, amena y de fácil acceso, dentro y fuera del país.

\section{Hacia un Paraguay plurilingüe}

En las últimas décadas, en Paraguay se han dado cambios muy significativos en la política relativa a las lenguas. De una política de imposición del castellano como lengua única, hemos pasado a otra que promueve el bilingüismo guaranícastellano, la defensa y preservación de las lenguas indígenas, el aseguramiento y el respeto de la lengua de señas y la promoción de la enseñanza de las lenguas extranjeras. La Constitución Nacional de 1992 oficializó la lengua guaraní junto al castellano. La Ley $4251 / 10$ de Lenguas creó la Secretaría de Políticas Lingüísticas y la Academia de la Lengua Guaraní, y estableció las políticas a ser encaradas desde el Estado en forma conjunta con la sociedad civil. La mencionada normativa, en su Art. 3으, de las lenguas oficiales, establece que las mismas tendrán vigencia y uso en los tres Poderes del Estado y en todas las instituciones públicas, al tiempo de obligar al Estado a prestar especial atención a la lengua guaraní, como signo de identidad cultural de la nación, instrumento de cohesión nacional, y medio de comunicación de la mayoría de la población paraguaya.

La lengua guaraní es la lengua propia del Paraguay, es la lengua que identifica y distingue al Paraguay de las demás naciones. Pese a ello, ha sido negada por el Estado por largas décadas y siglos. "Ninguneando una lengua ninguneamos a sus hablantes; ninguneando el guaraní ninguneamos al Paraguay", afirmaba el Dr. Rubén Martínez Huelmo, presidente del Parlamento del MERCOSUR (Parlasur), en la ocasión de la firma del convenio de cooperación entre dicho Parlamento y la Secretaría de Políticas Lingüísticas, el 7 de marzo del presente año, para la selección de traductores guaraní-castellano, guaraní-portugués, que oficiarán de intérpretes en las sesiones del Parlasur.

El castellano también es lengua oficial del Paraguay, y también merece atención del Estado. Por suerte, esta lengua goza de buena salud; la misma es - hasta ahora - la única lengua de trabajo del Estado paraguayo, en gran medida lengua única del sistema educativo, de los medios de comunicación, de las instituciones de la sociedad civil; el mismo goza de mucho prestigio, dadas las políticas desarrolladas a su favor desde el Estado. El guaraní es el idioma oficial con desventajas; el mismo no tiene aún igual estatus que el castellano ni recibe desde el Estado las mismas políticas favorables que recibe la lengua de Cervantes. El guaraní tiene muy poca presencia escrita y muy poco visualizado en la sociedad. Es por ello que la Ley de Lenguas establece refuerzos positivos hacia ella. Su uso irá normalizándose en la administración del Estado, y en otros 
ámbitos no habituales de uso. También está ocupando espacios muy importantes a nivel internacional, como en el Parlamento del MERCOSUR, donde es lengua de trabajo junto al castellano y portugués. Es, además, lengua oficial en algunas provincias de Argentina; es enseñado en las universidades más prestigiosas del mundo. Con las nuevas políticas lingüísticas paraguayas y las acciones que se desprenden de ellas, estoy seguro de que el jardín lingüístico paraguayo estará asegurado.

\section{Nota}

1 - El Censo Nacional de Población y Vivienda de 2012 lamentablemente no arrojará resultados con respecto al uso de las lenguas en el país, por la insuficiencia de informaciones lingüísticas que fueron recogidas.

\section{Referencias}

GONZÁLEZ TORRES, Dionisio. Cultura Guaraní. Asunción, Paraguay, 2007.

MELIÀ, Bartomeu. Pasado, presente y futuro de la lengua guaraní. Imprenta Salesiana. Asunción, Paraguay, 2010.

ROMERO, Roberto A. Protagonismo histórico de la lengua guaraní. 2da. Edición, Arte Final, Asunción, Paraguay, 1998.

\section{RESUMEN}

Paraguay es un crisol lingüístico desde su mismo inicio. Además de las dos lenguas oficiales, el guaraní y el castellano, los 19 pueblos indígenas usan sus lenguas propias, y las distintas comunidades de inmigrantes también mantienen sus respectivos idiomas. La lengua históricamente mayoritaria ha sido y sigue siendo el guaraní; pese a ello, desde el Estado y la sociedad en general siempre se lo ha subvalorado. Actualmente, cerca del $90 \%$ de la población habla esta lengua, y el $70 \%$, el castellano.

El guaraní es la lengua indígena más vigorosa del continente. Es idioma oficial del Paraguay; es una lengua hablada por una población criolla en todo el territorio paraguayo; es también lengua del MERCOSUR y lengua de trabajo del Parlasur. Actualmente es hablada por más de 5.000.000 de personas dentro del territorio paraguayo, y también por la misma cantidad fuera del mismo, especialmente en los países del MERCOSUR. Como ocurre en todo proceso migratorio, los paraguayos que dejan sus tierras para vivir en otros países llevan consigo sus lenguas. Esto ocurre, por ejemplo, con compatriotas que migran a San Pablo; ellos siguen hablando entre sí la lengua que les transmitieron sus padres en el calor del hogar: el guaraní. Empero, entre los migrantes paraguayos - así como ocurre en la sociedad paraguaya - se presenta un fenómeno muy peligroso para la vida futura de la lengua guaraní: el corte generacional. La transmisión de padres a hijos es condición necesaria para la longevidad de una lengua. En los últimos años ha habido avances muy significativos en la forma de gestionar las lenguas en Paraguay. Con las nuevas políticas lingüísticas desplegadas y las acciones que se desprenden de ellas, podemos estimar que estará asegurado el jardín lingüístico paraguayo.

Palabras clave: lengua guaraní; lenguas oficiales; migrantes. 


\begin{abstract}
From its very beginning, Paraguay has been a linguistic melting pot. Besides its two official languages - Guaraní and Spanish - there are 19 aboriginal communities that use their own native language, as well as a variety of immigrant groups that keep their language alive. Guaraní has always been - until now - the communication means of the majority; however, both the State and the general society have underestimated it. At this moment, nearly $90 \%$ of the Paraguayan population speaks Guaraní, and 70\% speaks Spanish. The Guaraní language is the most vigorous of the American continent. It is the official language of Paraguay; it is spoken by the creole population in all the Paraguayan territory; it is also one of the languages of MERCOSUR and one of the working languages of PARLASUR. Nowadays, Guaraní is spoken by more than 5 million people inside the Paraguayan territory and the same quantity abroad, especially in MERCOSUR countries. As normally happens in every migration process, Paraguayan people that leave their country to live abroad take their language with them. This happens, for example, with the compatriots that migrate to São Paulo; they keep on speaking the language that was transmitted by their parents at home: Guaraní. Nevertheless, between Paraguayan immigrants - as it happens inside the Paraguayan society - a very dangerous phenomenon threatens the future of the Guaraní language: the generation gap. The transmission of the language from parent to child is a required condition for the longevity of a language. In the past few years there have been some very significant advances in the management of the Paraguayan languages. With the spread of the new linguistic policies and the actions that follow them, we can foresee the safety of the well-named "Paraguayan linguistic garden".
\end{abstract}

Keywords: official languages; guaraní language; migrants. 